\title{
Utility of 4-Aryl-8-arylidene-3, 4, 5, 6, 7, 8-hexa- hydroquinazoline-2-thiol for the Synthesis of Some New Heterocycles as Antioxidant Agents
}

\author{
NADIA .T.A. DAWOUD \\ Department of Chemistry, Faculty of Science "Girls", \\ Al-Azhar University, Nasr City, Cairo, Egypt \\ dawoudnadia@yahoo.com
}

Received 6 November 2016 / Accepted 26 November 2016

\begin{abstract}
The present study deals with the synthesis and reactions of 4-aryl-8-arylidene-3,4,5,6,7,8hexahydroquinazoline-2-thiol (1). Hydrazinolysis of compound $\mathbf{1}$ yielded hexahydroquinazolinyl hydrazine 2. Acetylation of compound 2 yielded $[1,2,4]$ triazolo[3,4-b] quinazoline derivative $\mathbf{3}$. The reaction of compound $\mathbf{2}$ with diethyl oxalate and ethyl acetoacetate afforded hexahydro quinazolin-2yl-hydrazinyl-2-oxoacetate 9 and hexahydroquinazolin-2-yl-3-oxobutane hydrazide 10. Treatment of compound $\mathbf{1}$ with chloroacetyl chloride gave tetrahydro-2H-thiazolo [2,3-b] quinazolin-3(5H)-one $\mathbf{1 3}$ which was used as a key for the synthesis of a series of heterocyclic compounds (14-19). The structures of the newly synthesized compounds were elucidated on the basis of elemental analysis, spectral data. The antioxidant activities of some new synthesized compounds have been screened. Compounds $2,3,12,13,15,16$ and 19 showed antioxidant activity using the DPPH method.
\end{abstract}

Keywords: Quinazoline-2-thiols, Triazolo[3,4- $b$ ] quinazoline derivatives, Thiazolo[2,3- $b$ ] quinazoline derivatives, Antioxidant activity

\section{Introduction}

Owing to the significant biological activities, quinazoline derivatives have drawn more and more attention in the synthesis and bioactivities research. Quinazoline derivatives, which belong to the $N$-containing heterocyclic compounds, have caused universal concerns due to their biopharmaceutical activities. Researchers have already determined many therapeutic activities of quinazoline derivatives, including anticancer ${ }^{1-4}$, antiinflammatory ${ }^{5,6}$, antibacterial ${ }^{7-}$ ${ }^{10}$, analgesic ${ }^{5,9}$, antiviral ${ }^{11}$, anticytotoxic ${ }^{12}$, antispasm ${ }^{9,13}$, antituberculosis ${ }^{14}$, antioxidant ${ }^{15}$, antimalarial $^{16}$, antihypertension ${ }^{17}$, antiobesity ${ }^{18}$, antipsychotic ${ }^{19}$, anti-diabetics ${ }^{20}$, etc. Medicinal chemists synthesized quinazoline derivatives with different biological activities by installing various active groups to the quinazoline moiety. Herein, we report the design and synthesis of novel quinazoline derivatives bearing a biologically active, thiazole, pyrazole, triazole, pyran, pyridine, pyrimidine, triazine and triazepine moieties for evaluation to antioxidant activities. 


\section{Experimental}

All melting points for the prepared derivatives were measured in capillary tubes using a Gallen-Kamp apparatus and were uncorrected. The FT-IR spectra were recorded on a Perkin-Elmer 1650 spectrophotometer ( $\mathrm{KBr}$ pellets). The ${ }^{1} \mathrm{H},{ }^{13} \mathrm{C}$ NMR spectra were measured in dimethylsulphoxide- $d 6$ as a solvent using a Varian Gemini 180 spectrometer operating at $400 \mathrm{MHz}$ for ${ }^{1} \mathrm{H}$ and $75 \mathrm{MHz}$ for ${ }^{13} \mathrm{C}$ NMR. TMS was used as an internal standard and the chemical shifts were reported as $\delta \mathrm{ppm}$. The FAB mass spectra were recorded on a JEOL SX 102/DA-6000 mass spectrometer.

Synthesis of 1-(8-arylidene-4-phenyl-3, 4, 5, 6, 7, 8-hexahydroquinazolin-2-yl)hydrazine (2)

To ethanolic solution of compound $1 \mathbf{a}(0.01 \mathrm{~mol})$, hydrazine hydrate $(0.01 \mathrm{~mol})$ was added slowly. The reaction mixture was heated under reflux for $18 \mathrm{~h}$ or for the time needed to complete the reaction. The solid obtained was recrystallized from ethanol to give compound 2.

$\mathrm{C}_{21} \mathrm{H}_{22} \mathrm{~N}_{4}$ (MW330): pale yellow crystals; $80 \%$ yield; m. p $165-167{ }^{\circ} \mathrm{C}$. Anal Calc: C, 76.33; H, 6.71; N, 16.96; Found: C, 76.25; H, 6.58; N, 16.74. FT-IR $\left(\mathrm{KBr} \mathrm{cm}^{-1}\right)$ v max: 3411, 3338, $3285\left(\mathrm{NH}, \mathrm{NH}_{2}\right) ; 1627(\mathrm{C}=\mathrm{N}) ; 2930,2857\left(\mathrm{CH}\right.$-aliphatic); 3057, 3026(CH-aromatic). ${ }^{1} \mathrm{H}$ NMR $(\delta$ ppm) $($ DMSO- $d 6): \delta=10.2(\mathrm{~s}, 1 \mathrm{H}, \mathrm{NH}) ; 8.7(\mathrm{~s}, 1 \mathrm{H}, \mathrm{NH}) ; 7.2(\mathrm{~s}, 1 \mathrm{H}, \mathrm{CH}=\mathrm{C}) ; 7.2-7.9(\mathrm{~m}$ $, 10 \mathrm{H}, \mathrm{Ar}-\mathrm{H}) ; 8.7$ (s,br,2H, $\left.\mathrm{NH}_{2}\right) ; 5.7(\mathrm{~s}, 1 \mathrm{H}, \mathrm{CH}$ pyrimidine $) ; 1.2-2.5\left(\mathrm{~m}, 6 \mathrm{H}, 3 \times \mathrm{CH}_{2}\right) . \mathrm{MS}: \mathrm{m} / \mathrm{z}(\%)$ $330\left(1 \mathrm{M}^{+}\right)$; $331\left(0.7 \mathrm{M}^{+}+1\right)$; $332\left(1 \mathrm{M}^{+}+2\right) ; 333\left(0.7 \mathrm{M}^{+}+3\right)$; $91(100)$.

Synthesis of 1-(9-arylidene-3-methyl-5-phenyl-6,7,8,9-tetrahydro[1,2,4]triazolo[3,4-b] quinazolin -1(5H)-yl)ethanone (3)

A mixture of compound $2(0.01 \mathrm{~mol})$ and acetic anhydride $(10 \mathrm{~mL})$ was heated under reflux for $10 \mathrm{~h}$ on water bath, cooled to room-temp, poured onto ice/cold water. The precipitate was collected, filtered and recrystallized from ethanol to give compound $\mathbf{3}$.

$\mathrm{C}_{21} \mathrm{H}_{20} \mathrm{~N}_{2} \mathrm{~S}$ (MW332); brown powder; 60\% yield; m.p143-145 ${ }^{\circ} \mathrm{C}$. Anal Calc: C, 75.87; H, 6.06; N, 8.43; S, 9.64; Found: C, 75.65; H, 5.93; N, 8.35; S, 9.56. FT-IR $\left(\mathrm{KBr} \mathrm{cm}^{-1}\right)$

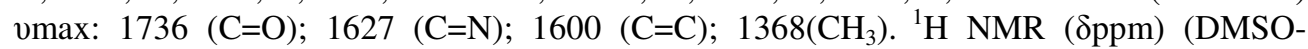
$d 6): \delta=7.1-7.9(\mathrm{~m}, 10 \mathrm{H}, \mathrm{Ar}-\mathrm{H}) ; 6.6(\mathrm{~s}, 1 \mathrm{H}, \mathrm{CH}=\mathrm{C}) ; 5.9(\mathrm{~s}, 1 \mathrm{H}, \mathrm{CH}$ pyrimidine $) ; 2.5(\mathrm{~s}, 3 \mathrm{H}$, $\left.\mathrm{COCH}_{3}\right) ; 1.2\left(\mathrm{~s}, 3 \mathrm{H}, \mathrm{CH}_{3}\right) ; 1.7-2.8\left(\mathrm{~m}, 6 \mathrm{H}, 3 \times \mathrm{CH}_{2}\right)$

Synthesis of 1-(1-(9-arylidene-3-methyl-5-phenyl-6,7,8,9-tetrahydro[1,2,4]triazolo[3,4-b] quinazolin-1 $(5 \mathrm{H})$-yl)ethylidene)thiosemicarbazide (4)

A mixture of compound $3(0.01 \mathrm{~mol})$ and thiosemicabazide $(0.01 \mathrm{~mol})$ in $(30 \mathrm{~mL})$ ethanol was heated under reflux for $10 \mathrm{~h}$ in the presence of sodium ethoxide (prepared from $1 \mathrm{~g} \mathrm{Na}$ in $20 \mathrm{~mL} \mathrm{EtOH}$ ), cooled to room-temp, poured onto ice cold water. The solid was filtered and recrystallized from methanol to give compound 4.

$\mathrm{C}_{26} \mathrm{H}_{27} \mathrm{~N}_{7} \mathrm{~S}$ (MW469); pale yellow powder; $70 \%$ yield; m. p. $178-180{ }^{\circ} \mathrm{C}$. Anal Calc: $\mathrm{C}$, $66.50 ; \mathrm{H}, 5.80 ; \mathrm{N}, 20.88 ; \mathrm{S}, 6.83$. Found: C, 66.33; H, 5.78; N, 20.76; S, 6.71. FT-IR (KBr $\left.\mathrm{cm}^{-1}\right)$ vmax: $342,3235,3125\left(\mathrm{NHNH}_{2}\right) ; 1640(\mathrm{C}=\mathrm{N}) ; 1592(\mathrm{C}=\mathrm{C}) ; 1274(\mathrm{C}=\mathrm{S}) .{ }^{1} \mathrm{HNMR}(\delta$ ppm) (DMSO-d6): $\delta=8.3(\mathrm{~s}, 1 \mathrm{H}, \mathrm{NH}) ; 7.07 .7(\mathrm{~m}, 10 \mathrm{H}, \mathrm{ArH}) ; 4.6\left(\mathrm{~s}, \mathrm{br}, 2 \mathrm{H}, \mathrm{NH}_{2}\right) ; 5.4(\mathrm{~s}, 1 \mathrm{H}, \mathrm{CH}$ pyrimidine); $1.1\left(\mathrm{~s}, 3 \mathrm{H}, \mathrm{CH}_{3}\right) ; 1.2\left(\mathrm{~s}, 3 \mathrm{H}, \mathrm{CH}_{3}\right) ; 1.8-2.5\left(\mathrm{~m}, 6 \mathrm{H}, 3 \mathrm{xCH}_{2}\right)$.

Synthesis of 1-(1-(9-arylidene-3-methyl-5-phenyl-6,7,8,9-tetrahydro-[1,2,4]triazolo[3,4-b] quinazolin-1(5H)-yl)ethylidene)thiosemicarbazide Schiff's base (5)

To ethanolic solution of compound 4 ( $0.01 \mathrm{~mol})$, 2-chlorobenzaldehyde $(0.01 \mathrm{~mol})$ was added. 
The reaction mixture was heated under reflux for $8 \mathrm{~h}$ in the presence of catalytic amount of acetic acid, cooled to room-temp. The solid separated was filtered, recrytstallized from ethanol to give Schiff's base $\mathbf{5}$.

$\mathrm{C}_{33} \mathrm{H}_{30} \mathrm{ClN}_{7} \mathrm{~S}$ (MW 591.5); yellow powder; $75 \%$ yield; m.p. $1185-187{ }^{\circ} \mathrm{C}$. Anal Calc: C, 66.93; H, 5.11; Cl, 5.99; N, 16.56; S, 5.41. Found: C, 66.75; H, 5.00; Cl, 5.67; N, 16.33; S, 5.15. FT-IR (KBr) vmax: $3248(\mathrm{NH}) ; 1117(\mathrm{C}=\mathrm{S}) ; 611(\mathrm{C}-\mathrm{Cl})$. MS: m/z (\%) $591.5\left(2 \mathrm{M}^{+}\right) ; 60$ (100).

Synthesisof1-(9-arylidene-3-methyl-5-phenyl-6,7,8,9-tetrahydro-[1,2,4]triazolo[3,4b] quinazolin -1(5H)-yl)-3-(2-chlorophenyl)prop-2-en-1-one (6)

A mixture of compound $3(0.01 \mathrm{~mol}), 2$-chlorobenzaldehyde $(0.01 \mathrm{~mol})$ in $(30 \mathrm{~mL})$ ethanol was heated under reflux for $6-8 \mathrm{~h}$ in the presence of $20 \% \mathrm{KOH}$, cooled to room-temp, poured onto $\mathrm{H}_{2} \mathrm{O} / \mathrm{HCl}$ mixture. The solid separated was filtered and recrystallized from ethanol to give compound $\mathbf{6}$.

$\mathrm{C}_{32} \mathrm{H}_{27} \mathrm{ClN}_{4} \mathrm{O}$ (MW519); brownish red crystals; $75 \%$ yield; m.p168-170 ${ }^{\circ} \mathrm{C}$. Anal Calc: C, 74.05; H, 5.24; Cl, 6.83; N, 10.79. Found: C, 73.65; H, 4.93; Cl, 6.65; N, 10.35. FT-IR $(\mathrm{KBr})$ vmax: $1667(\mathrm{C}=\mathrm{O}) ; 1600(\mathrm{C}=\mathrm{N}) ; 689(\mathrm{C}-\mathrm{Cl}) .{ }^{1} \mathrm{HNMR}(\delta \mathrm{ppm})(\mathrm{DMSO}-d 6): \delta=7.2-8.0$ $(\mathrm{m}, 14 \mathrm{H}, \mathrm{Ar}-\mathrm{H}) ; 7.10 \quad(\mathrm{~s}, 1 \mathrm{H}, \mathrm{CH}=\mathrm{C}) ; 6.6 \quad(\mathrm{~d}, 1 \mathrm{H}, \mathrm{CH}=\mathrm{CHCO}) ; 7.8 \quad(\mathrm{~d}, 1 \mathrm{H}, \mathrm{CH}=\mathrm{CHCO}) ; 5.7$ (s,1H,CH pyrimidine) ; 1.7-2.8 (m,6H,3 $\left.\mathrm{xCH}_{2}\right) ; 1.3\left(\mathrm{~s}, 3 \mathrm{H}, \mathrm{CH}_{3}\right)$.

Synthesis of 3-(9-arylidene-3-methyl-5-phenyl-6,7,8,9-tetrahydro-

[1,2,4] triazolo[3,4-b] quinazolin -1(5H)-yl)-5-(2-chlorophenyl)-4,5dihydropyrazole-1-carbothioamide (7)

A mixture of compound $6(0.01 \mathrm{~mol})$ and thiosemicarbazide $(0.01 \mathrm{~mol})$ in $(30 \mathrm{~mL})$ of ethanol was heated under reflux for $8 \mathrm{~h}$, cooled to room-temp. The solid separated was filtered and recrystallized from acetic acid to give compound 7.

$\mathrm{C}_{33} \mathrm{H}_{30} \mathrm{ClN}_{7} \mathrm{~S}$ (M W 591); orange crystals; $70 \%$ yield; m.p180-182 ${ }^{\circ} \mathrm{C}$. Anal Calc: C, 66.93; H, 5.11; Cl, 5.99; N, 16.56; S, 5.41. Found: C, 66.71; H, 5.00; Cl, 5.68; N, 16.34; S, 5.22. FT-IR (KBr) vmax: 3421, 3255( $\left.\mathrm{NH}_{2}\right) ; 1274(\mathrm{C}=\mathrm{S})$; 689(C-Cl). ${ }^{1} \mathrm{H}$ NMR $(\delta \mathrm{ppm})(\mathrm{DMSO}-$ $d 6): \delta=7.0-7.7(\mathrm{~m}, 14 \mathrm{H}, \mathrm{Ar}-\mathrm{H}) ; 6.7(\mathrm{~s}, 1 \mathrm{H}, \mathrm{CH}=\mathrm{C}) ; 5.4(\mathrm{~s}, 1 \mathrm{H}, \mathrm{CH}-$ pyrimidine $) ; 4.6\left(\mathrm{~s}, 2 \mathrm{H}, \mathrm{NH}_{2}\right)$; 4.3 (t,1H-Pyrazoline); 3.3 (dd,2H , $\mathrm{CH}_{2}$-pyrazoline ); 0.9 (s,3H, $\left.\mathrm{CH}_{3}\right)$.

Synthesis of 3-amino-6-(9-arylidene-3-methyl-5-phenyl-6, 7,8,9-tetrahydro[1,2,4]triazolo[3,4-b] quinazolin-1 $(5 \mathrm{H})$-yl)-4-(4-chlorophenyl)picolinonitrile (8)

A mixture of Compound $3(0.01 \mathrm{~mol})$, 2-chlorobenzaldehyde $(0.01 \mathrm{~mol})$, malononitrile $(0.01$ mol) and ammonium acetate $(0.01 \mathrm{~mol})$ in alcoholic solution of $\mathrm{NaOH}$ was heated under reflux for $6 \mathrm{~h}$, cooled to room -temp, neutralized with dil $\mathrm{HCl}$ until precipitation. The product was filtered, collected and recrystallized from ethanol to give compound 8 .

$\mathrm{C}_{35} \mathrm{H}_{28} \mathrm{ClN}_{7}$ (M W 581.5); brown powder; 70\% yield; m.p170-172 ${ }^{\circ} \mathrm{C}$. Anal Calc: C, 72.22; H, 4.85; Cl, 6.09; N, 16.84. Found: C, 72.12; H, 4.73; Cl, 5.65; N, 16.52. FT-IR $\left(\mathrm{KBr} \mathrm{cm}^{-1}\right) v$ $\max 3248,3155\left(\mathrm{NH}_{2}\right) ; 2204(\mathrm{C}=\mathrm{N}) ; 1639(\mathrm{C}=\mathrm{N})$. ${ }^{1} \mathrm{HNMR}(\delta \mathrm{ppm})(\mathrm{DMSO}-d 6): \delta=7.1-7.6$ (m,15H,Ar-H);6.9 (s,1H,CH=C); 5.4 (s,1H,CH-pyrimidine); $4.3\left(\mathrm{~s}, 2 \mathrm{H}, \mathrm{NH}_{2}\right) ; 0.9\left(\mathrm{~s}, 1 \mathrm{H}^{2} \mathrm{CH}_{3}\right) ; 1.4-$ $2.7\left(\mathrm{~m}, 6 \mathrm{H}, 3 \times \mathrm{CH}_{2}\right)$. MS: $m / z$ :(\%) $581\left(5 \mathrm{M}^{+}\right) ; 583\left(3 \mathrm{M}^{+}+2\right) ; 584\left(2 \mathrm{M}^{+}+3\right) ; 164$ (100).

Synthesis of ethyl 2-(2-(8-arylidene-4-phenyl-3,4,5,6,7,8-hexahydroquinazolin-2yl)hydrazin- yl) -2-oxoacetate (9)

A mixture of $2(0.01 \mathrm{~mol})$ and diethyl oxalate $(0.01 \mathrm{~mol})$ in $(25 \mathrm{~mL})$ ethanol was heated under reflux for $8 \mathrm{hrs}$. The solid separated was filtered, collected and recrystallized from 2:1 ethanol $/ \mathrm{H}_{2} \mathrm{O}$ mixture to give 9 . 
$\mathrm{C}_{25} \mathrm{H}_{26} \mathrm{~N}_{4} \mathrm{O}_{3}$ (M W 430); beige powder; 80\% yield; m.p150-152 ${ }^{\circ} \mathrm{C}$. Anal Calc: C, 69.75; $\mathrm{H}, 6.09 ; \mathrm{N}, 13.01$. Found: C, 69.73; H, 5.79; N, 12.91. FT-IR $\left(\mathrm{KBr} \mathrm{cm}^{-1}\right)$ vmax: $3215(\mathrm{NH})$; $1741(\mathrm{C}=\mathrm{O}) ; 1672(\mathrm{C}=\mathrm{O}) .{ }^{1} \mathrm{H}$ NMR $(\delta \mathrm{ppm})(\mathrm{DMSO}-d 6): \delta=14.2(\mathrm{~s}, 1 \mathrm{H}, \mathrm{NH}) ; 12.4(\mathrm{~s}, 1 \mathrm{H}, \mathrm{NH})$; $10.0(\mathrm{~s}, 1 \mathrm{H}, \mathrm{NH}) ; 7.2-7.9(\mathrm{~m}, 10 \mathrm{H}, \mathrm{Ar}-\mathrm{H}) ; 6.9(\mathrm{~s}, 1 \mathrm{H}, \mathrm{CH}=\mathrm{C}) ; 5.4$ (s,1H,CHpyrimidine); 4.2 $\left(\mathrm{q}, 2 \mathrm{H}, \mathrm{CH}_{2} \mathrm{CH}_{3}\right) ; 1.4-2.7\left(\mathrm{~m}, 6 \mathrm{H}, 3 \mathrm{xCH}_{2}\right) ; 1.0\left(\mathrm{t}, 3 \mathrm{H}, \mathrm{CH}_{2} \mathrm{CH}_{3}\right) .{ }^{13} \mathrm{C}$ NMR (400MHz, DMSOd6): $15.6\left(\mathrm{CH}_{3}\right) ; 63.8\left(\mathrm{CH}_{2}\right) ; 56.5(\mathrm{CH}-\mathrm{Pyrimidine}) ; 125.3-136.4$ (aromatic $>\mathrm{C}=\mathrm{C}<$ ); 122.0, 137.3 (CH=C-benzylidine );163.9, $165.2(2 \mathrm{C}=\mathrm{O}) ; 160.1(\mathrm{C}-\mathrm{NH}-\mathrm{NH})$.

Synthesis of 3-(8-arylidene-4-phenyl-3,4,5,6,7,8-hexahydroquinazolin-2-yl)-3oxobutane hydrazide (10)

A mixture of compound $2(0.01 \mathrm{~mol})$ and ethyacetoacetate $(0.01 \mathrm{~mol})$ in $(20 \mathrm{~mL})$ ethanol was heated under reflux for $6 \mathrm{~h}$. The solid separated was filtered and recrystallized from 1:1 ethanol $/ \mathrm{H}_{2} \mathrm{O}$ mixture to give compound $\mathbf{1 0}$.

$\mathrm{C}_{25} \mathrm{H}_{26} \mathrm{~N}_{4} \mathrm{O}_{2}$ (M W 414); yellow powder; $60 \%$ yield; m.p163-165 ${ }^{\circ} \mathrm{C}$. Anal Calc: $\mathrm{C}$, 72.44; H, 6.32; N, 13.52. Found: C, 72.23; H, 6.02; N, 13.35. FT-IR $\left(\mathrm{KBr} \mathrm{cm}^{-1}\right)$ vmax: 3372 $(\mathrm{NH}) ; 3232(\mathrm{NH}) ; 1716(\mathrm{C}=\mathrm{O}) ; 1636(\mathrm{C}=\mathrm{N}) .{ }^{1} \mathrm{H}$ NMR $(\delta \mathrm{ppm})(\mathrm{DMSO}-\mathrm{d} 6): \delta=12.0$ $(\mathrm{s}, 1 \mathrm{H}, \mathrm{NH}) ; 10.5$ (s,1H, NH);7.0 -7.7 (m,10H,Ar-H); $5.2 \quad$ (s,1H,CH-pyrimidine); 4.8 $\left(\mathrm{q}, 2 \mathrm{H}, \mathrm{CH}_{2} \mathrm{CH}_{3}\right) ; 3.9\left(\mathrm{~s}, 2 \mathrm{H}, \mathrm{CH}_{2}\right) ; 1.4\left(\mathrm{t}, 3 \mathrm{H}, \mathrm{CH}_{2} \mathrm{CH}_{3}\right) ; 1.2\left(\mathrm{~s}, 3 \mathrm{H}, \mathrm{CH}_{3}\right) ; 1.7-2.9\left(\mathrm{~m}, 6 \mathrm{H}, 3 \mathrm{xCH}_{2}\right)$.

Synthesis of 9-arylidene-5-phenyl-1,2,6,7,8,9-hexahydro-[1,2,4]triazolo[3,4b]quinazoline-3(5H)-thione (11)

A mixture of compound $2(0.01 \mathrm{~mol})$ and carbon disulphide $(0.01 \mathrm{~mol})$ in $(20 \mathrm{~mL})$ pyridine was heated under reflux for $8 \mathrm{~h}$, cooled to room-temp, poured onto ice $\mathrm{H}_{2} \mathrm{O} / \mathrm{HCl}$ mixture until precipitation. The solid separated was collected, filtered and recrystallized from ethanol to give compound 11.

$\mathrm{C}_{22} \mathrm{H}_{20} \mathrm{~N}_{4} \mathrm{~S}$ (M W 372); orange crystals; $85 \%$ yield; m.p170 ${ }^{\circ} \mathrm{C}$. Anal Calc: C, 70.94; H, 5.41; N, 15.04; S, 8.61. Found: C, 70.65; H, 5.21; N, 14.84; S, 8.35. FT-IR $\left(\mathrm{KBr} \mathrm{cm}^{-1}\right)$ vmax: $3216(\mathrm{NH}) ; 1267(\mathrm{C}=\mathrm{S}) .{ }^{1} \mathrm{H}$ NMR $(\delta \mathrm{ppm})(\mathrm{DMSO}-d 6): \delta=10.0(\mathrm{~s}, 1 \mathrm{H}, \mathrm{NH}) ; 8.7(\mathrm{~s}, 1 \mathrm{H}, \mathrm{NH})$; 7.2-7.7 (m,10Ar-H); $7.1(\mathrm{~s}, 1 \mathrm{H}, \mathrm{CH}=\mathrm{C}) ; 5.7(\mathrm{~s}, 1 \mathrm{H}, \mathrm{CH}$ pyrimidine $)$; $1.2-2.6\left(\mathrm{~m}, 6 \mathrm{H}, 3 \mathrm{xCH}_{2}\right)$.

Synthesis of 3-amino-11-arylidene-5-(2-chlorophenyl)-7-phenyl-1,4,5,7,8,9,10,11octahydro- [1,2,4 ]triazepino[3,4-b]quinazoline-4-carbonitrile (12)

To ethanolic solution of compound $2(0.1 \mathrm{~mol})$ containing $(5 \mathrm{~mL})$ piperidine, 2chlorobenzaldehde $(0.01 \mathrm{~mol})$ and malononitrile $(0.01 \mathrm{~mol})$ were added. The reaction mixture was heated under reflux for $8 \mathrm{~h}$, cooled to room-temp, poured onto crushed ice containing conc. $\mathrm{HCl}$. The solid was filtered and recrysallized from to give compound $\mathbf{1 2}$.

$\mathrm{C}_{31} \mathrm{H}_{27} \mathrm{ClN}_{6}$ (MW 519.5); yellow crystals; $75 \%$ yield; m.p175-177 ${ }^{\circ} \mathrm{C}$. Anal Calc: C, 71.73; $\mathrm{H}, 5.24 ; \mathrm{Cl}, 6.83 ; \mathrm{N}, 16.19$. Found: $\mathrm{C}, 71.66 ; \mathrm{H}, 5.11 ; \mathrm{Cl}, 6.75 ; \mathrm{N}, 16.02 . \mathrm{IR}\left(\mathrm{KBr} \mathrm{cm}^{-1}\right)$ vmax: 3362, 3276, $3150\left(\mathrm{NHNH}_{2}\right) ; 2199(\mathrm{C}=\mathrm{N}) ; 1624(\mathrm{C}=\mathrm{N}) .{ }^{1} \mathrm{H}-\mathrm{NMR}(\delta \mathrm{ppm})(\mathrm{DMSO}-d 6): \delta=7.2-$ 7.7 (m,14Ar-H); 6.9 (s,1H,NH); 5.8 (s,1H,CH-pyrimidine); 3.2 (d,1H, CH-triazepine); 3.9 (d,1H, CH-triazepine); 4.6 (s,br,2H, $\left.\mathrm{NH}_{2}\right) ; 1.2-2.1\left(\mathrm{~m}, 6 \mathrm{H}, 3 \mathrm{xCH}_{2}\right)$. MS: $\mathrm{m} / z(\%) 519.5\left(3 \mathrm{M}^{+}\right) ; 257(100)$.

Synthesis of 9-arylidene-5-phenyl-6,7,8,9-tetrahydro-2H-thiazolo[2,3-b]quinazolin3(5H)-one (13)

A mixture of compound $1(0.01 \mathrm{~mol})$, chloroacetyl chloride $(0.01 \mathrm{~mol})$ in $(20 \mathrm{~mL})$ pyridine was heated under reflux for $6 \mathrm{~h}$, cooled to room-temp, poured onto $\mathrm{H}_{2} 0 / \mathrm{HCl}$ mixture. The solid was filtered, recrystallized from petroleum ether 60-80 to give compound $\mathbf{1 3}$. 
$\mathrm{C}_{23} \mathrm{H}_{20} \mathrm{~N}_{2} \mathrm{OS}$ (MW 372); brown crystals; 65\% yield; m.p150-152 ${ }^{\circ} \mathrm{C}$. Anal Calc: C, 74.16; H, 5.41; N, 7.52; S, 8.61. Found: C, 73.88; H, 5.26; N, 7.38; S, 8.54. FT-IR $\left(\mathrm{KBr} \mathrm{cm}^{-1}\right)$ vmax: $1712(\mathrm{C}=\mathrm{O}) .{ }^{1} \mathrm{H}$ NMR $(\delta \mathrm{ppm})(\mathrm{DMSO}-d 6): \delta=7.3-8.0(\mathrm{~m}, 10 \mathrm{H}, \mathrm{Ar}-\mathrm{H}) ; 5.8(\mathrm{~s}, 1 \mathrm{H}$, CH-pyrimidine); 4.3 (s, 2H, $\mathrm{CH}_{2} \mathrm{CO}$ ) ); 1.2-2.8 (m, 6H, $3 \mathrm{XCH}_{2}$ ).

Synthesis of ethyl 2-(9-arylidene-3-oxo-5-phenyl-3,5,6,7,8,9-hexahydro-2Hthiazolo[2,3-b] quinazolin-2-yl)-2-oxoacetate (14)

To a mixture of sodium ethoxide solution $(0.02 \mathrm{~mol})(0.46 \mathrm{mg}$ of sodium in $20 \mathrm{~mL}$ ethanol $)$ and diethyl oxalate $(0.02 \mathrm{~mol})$, compound $13(0.02 \mathrm{~mol})$ was added at $0{ }^{\circ} \mathrm{C}$. The reaction mixture was stirred at $0{ }^{\circ} \mathrm{C}$ for $1 \mathrm{~h}$, heated under reflux for another $3 \mathrm{~h}$, left at room temperature overnight. The solution was neutralized with ice-cold diluted $\mathrm{HCl}$. The mixture was extracted with diethyl ether and the organic layer was collected, washed with water, dried and crystallized from benzene to give compound $\mathbf{1 4}$.

$\mathrm{C}_{27} \mathrm{H}_{24} \mathrm{~N}_{2} \mathrm{O}_{4} \mathrm{~S}$ (MW 472); crystals; $60 \%$ yield; m.p148-150 ${ }^{\circ} \mathrm{C}$. Anal Calc: C, 68.62; H, 5.12; N, 5.93; S, 6.79. Found: C, 68.48; H, 5.06; N, 5.76; S, 6.55. FT-IR ( $\left.\mathrm{KBr} \mathrm{cm}^{-1}\right)$ vmax: $1728(\mathrm{C}=\mathrm{O}) ; 1628(\mathrm{C}=\mathrm{N}) .{ }^{1} \mathrm{H}$ NMR $(\delta \mathrm{ppm})(\mathrm{DMSO}-d 6): \delta=7.1-7.6(\mathrm{~m}, 10 \mathrm{Ar}-\mathrm{H}) ; 6.9$ $(\mathrm{s}, 1 \mathrm{H}, \mathrm{CH}=\mathrm{C}) ; 6.8$ (s,1H,CH-pyrimidine); 4.4 (s,1H,CH-thiazole); 4.1 (q, $\left.2 \mathrm{H}, \mathrm{CH}_{2} \mathrm{CH}_{3}\right) ; 1.1$ $\left(\mathrm{t}, 3 \mathrm{H}, \mathrm{CH}_{2} \mathrm{CH}_{3}\right) ; 1.6-2.9\left(\mathrm{~m}, 6 \mathrm{H}, 3 \times \mathrm{CH}_{2}\right)$. MS: $\mathrm{m} / z(\%) 472\left(6 \mathrm{M}^{+}\right) ; 125$ (100).

Synthesis of 9-arylidene-5-phenyl-6,7,8,9-tetrahydro-1H-pyrazolo[4,3-d]-5Hthiazolo[2,3-b]-3-carboxlate quinazoline derivative (15)

To ethanolic solution of compound $14(0.01 \mathrm{~mol})$, Hydrazine hydrate $(0.01 \mathrm{~mol})$ was added at $0{ }^{\circ} \mathrm{C}$. The reaction mixture was heated under reflux for $6 \mathrm{~h}$. The solid obtained was filtered and recrystallized from the cyclohexane to give $\mathbf{1 5}$.

$\mathrm{C}_{27} \mathrm{H}_{24} \mathrm{~N}_{4} \mathrm{O}_{2} \mathrm{~S}$ (MW 468); beige crystals; 70\% yield; m.p170-172 ${ }^{\circ} \mathrm{C}$. Anal Calc: C, 69.21; H, 5.16; N, 11.96; S, 6.84. Found: C, 69.00; H, 5.16; N, 11.67; S, 6.72. FT-IR (KBr $\mathrm{cm}^{-1}$ ) vmax: $3185(\mathrm{NH}) ; 1735(\mathrm{C}=\mathrm{O}) .{ }^{1} \mathrm{H}$ NMR $(\delta \mathrm{ppm})(\mathrm{DMSO}-d 6): \delta=8.1(\mathrm{~s}, 1 \mathrm{H}, \mathrm{NH}) ; 7.2-$ $7.6(\mathrm{~m}, 10 \mathrm{Ar}-\mathrm{H}) ; 6.7(\mathrm{~s}, 1 \mathrm{H}, \mathrm{CH}=\mathrm{C}) ; 5.7(\mathrm{~s}, 1 \mathrm{H}, \mathrm{CH}-$ pyrimidine $) ; 4.2\left(\mathrm{q}, 2 \mathrm{H}, \mathrm{CH}_{2} \mathrm{CH}_{3}\right) ; 1.2$ $\left(\mathrm{t}, 3 \mathrm{H}, \mathrm{CH}_{2} \underline{\mathrm{CH}}_{3}\right) ; 1.7-2.8\left(\mathrm{~m}, 6 \mathrm{H}, 3 \mathrm{xCH}_{2}\right)$. MS: $m / z(\%) 468\left(11 \mathrm{M}^{+}\right) ; 343$ (100).

Synthesis of 9-arylidene-5-phenyl-6,7,8,9-tetrahydro-1H-pyrazolo[4,3-d]-5Hthiazolo[2,3-b]-3-carbohydrazide quinazoline derivative (16)

To ethanolic solution of compound $14(0.01 \mathrm{~mol})$, excess of hydrazine hydrate $(0.02 \mathrm{~mol})$ was added at $0{ }^{\circ} \mathrm{C}$. The reaction mixture was heated under reflux for $8 \mathrm{~h}$. The solid obtained was filtered and recrystallized from ethanol to give compound $\mathbf{1 6 .}$

$\mathrm{C}_{25} \mathrm{H}_{22} \mathrm{~N}_{6} \mathrm{OS}$ (MW454); brownish red crystals; $70 \%$ yield; m.p185-1870 ${ }^{\circ} \mathrm{C}$. Anal Calc: C, 69.21; H, 5.16; N, 11.96; S, 6.84. Found: C, 69.00; H, 5.16; N, 11.67; S, 6.72. FT-IR $\left(\mathrm{KBr} \mathrm{cm}^{-1}\right)$ v max: $3431,3335,3215\left(\mathrm{NHNH}_{2}\right) ; 1636(\mathrm{C}=\mathrm{O}) .{ }^{1} \mathrm{H}$ NMR $(\delta \mathrm{ppm})(\mathrm{DMSO}-d 6)$ : $\delta=10.0(\mathrm{~s}, 1 \mathrm{H}, \mathrm{NH}) ; 9.9(\mathrm{~s}, 1 \mathrm{H}, \mathrm{NH}) ; 7.2-7.7 \quad(\mathrm{~m}, 10 \mathrm{Ar}-\mathrm{H}) ; 4.2 \quad\left(\mathrm{~s}, \mathrm{br}, 2 \mathrm{H}, \mathrm{NH}_{2}\right) ; 1.2-2.5$ $\left(\mathrm{m}, 6 \mathrm{H}, 3 \times \mathrm{xCH}_{2}\right)$. MS: $\mathrm{m} / z(\%) 455\left(2 \mathrm{M}^{+}+1\right) ; 439$ (100).

Synthesis of 2-(2-chlorobenzylidene)-9-arylidene-5-phenyl-6,7,8,9-tetrahydro-2Hthiazolo [2,3-b] quinazolin-3(5H)-one (17)

A mixture of compound $\mathbf{1 3}(0.01 \mathrm{~mol}), 2$-chlorobenzaldehyde $(0.01 \mathrm{~mol})$ was heated under reflux for $6 \mathrm{hrs}$ in the presence of $20 \% \mathrm{KOH}$, cooled to room-temp, poured onto $\mathrm{H}_{2} \mathrm{O} / \mathrm{HCl}$ mixture. The solid was collected, filtered and recrystallized from ethanol to give $\mathbf{1 7}$. 
$\mathrm{C}_{30} \mathrm{H}_{23} \mathrm{ClN}_{2} \mathrm{OS}$ (MW 494); orange crystals; $75 \%$ yield; m.p163-165 ${ }^{\circ} \mathrm{C}$. Anal Calc: C, 72.79; H, 4.68; Cl, 7.16; N, 5.66; S, 6.48. Found: C, 72.65; H, 4.47; Cl, 7.03; N, 5.54; S, 6.39.). FT-IR $\left(\mathrm{KBr} \mathrm{cm}{ }^{-1}\right)$ v max: $3431,3335,3215\left(\mathrm{NHNH}_{2}\right) ; 1675(\mathrm{C}=\mathrm{O}) .{ }^{1} \mathrm{H}$ NMR $(\delta$ ppm) (DMSO- $d 6): \delta=7.7$ (s, 1H, CH=CCO) 7.2-7.7 (m, 14H, Ar-H).

Synthesis of ethyl 3-(9-arylidene-3-oxo-5-phenyl-3,5,6,7,8,9-hexahydro-2Hthiazolo[2,3-b] quinazolin-2-yl)-3-(2-chlorophenyl)-2-cyanopropanoate (18a) and 2-((9-arylidene-3-oxo-5-phenyl-3,5, 6,7,8,9-hexahydro-2H-thiazolo[2,3b]quinazolin-2-yl)(2-chlorophenyl)methyl) malononitrile (18b)

A mixture of compound $17(0.01 \mathrm{~mol})$, active methylene compounds namely, ethyl cyanoacetate and/or malononitrile $(0.01 \mathrm{~mol})$ in $(20 \mathrm{~mL})$ acetone, and potassium carbonate $(0.01 \mathrm{~mol})$ was cooled to $5-10{ }^{\circ} \mathrm{C}$ and stirred for $3-5 \mathrm{~h}$ maintaining the same temperature. The reaction mixture poured onto water. The solid was filtered and recrysallized from ethanol to give 18a, $\mathbf{b}$.

18a: $\mathrm{C}_{35} \mathrm{H}_{30} \mathrm{ClN}_{3} \mathrm{O}_{3} \mathrm{~S}$ (MW 607.5) brownish red; 65\% yield; m.p163-165 ${ }^{\circ} \mathrm{C}$. Anal Calc: C, 69.12; H, 4.97; Cl, 5.83; N, 6.91; S, 5.27. Found: C, 69.00; H, 4.76; Cl, 5.73; N, 6.70; S, 5.06. FT-IR $\left(\mathrm{KBr} \mathrm{cm}^{-1}\right)$ v max: $2201(\mathrm{CN}) ; 1666(\mathrm{C}=\mathrm{O}) ; 698(\mathrm{C}-\mathrm{Cl}) .{ }^{1} \mathrm{H}$ NMR $(\delta \mathrm{ppm})$ (DMSO-d6): $\delta=7.1-7.7(\mathrm{~m}, 14 \mathrm{H}, \mathrm{Ar}-\mathrm{H}) ; 3.8$ (d, 1H, CH-thiazole); 3.9 (t, 1H, CHCH); 3.7 (d, 1H, CHCH.CN .COOEt).

18b: $\mathrm{C}_{33} \mathrm{H}_{25} \mathrm{ClN}_{4} \mathrm{OS}$ (MW 560.5) brown powder; 75\% yield; m.p165-167 ${ }^{\circ} \mathrm{C}$. Anal Calc: C, 70.64; H, 4.49; Cl, 6.32; N, 9.99; S, 5.71. Found: C, 70.55; H, 4.27; Cl, 6.22; N, 9.76; S, 5.56. FT-IR $\left(\mathrm{KBr} \mathrm{cm}^{-1}\right)$ v max: $2209(\mathrm{C}=\mathrm{N}) ; 697(\mathrm{C}-\mathrm{Cl}) .{ }^{1} \mathrm{H}$ NMR $(\delta \mathrm{ppm})$ (DMSO-d6): $\delta=7.1-7.7(\mathrm{~m}, 14 \mathrm{H}, \mathrm{Ar}-\mathrm{H}) ; 3.8$ (d,1H,CH-thiazole); $3.2(\mathrm{t}, 1 \mathrm{H}, \underline{\mathrm{CHCHCN}}) ; 3.8\left(\mathrm{~d}, 1 \mathrm{H}, \mathrm{CH}(\mathrm{CN})_{2}\right)$.

Synthesis of 2,4-diamino- Synthesis of 5-(8-arylidene-4-phenyl-3,4,5,6,7,8hexahydroquinazo- lin-2-yl-thio)-5H-chromeno [2,3-b] pyridine -3-carbonitrile (19)

A mixture of compound $1 \mathrm{a}(0.01 \mathrm{~mol}), 2,4$-dihdroxybenzaldehyde $(0.01 \mathrm{~mol})$, malononitrile $(0.02 \mathrm{~mol})$ were dissolved in $(2 \mathrm{~mL})$ ethanol and $20 \mathrm{~mL}$ of pyridine was stirred under reflux for 2-6 h. The reaction mixture was cooled to room temperature, neutralized with dil $\mathrm{HCl}$ until precipitation, filtered off, washed with water and recrystallized from $1: 1$ ethanol/water mixture to give 19.

$\mathrm{C}_{34} \mathrm{H}_{28} \mathrm{~N}_{6} \mathrm{O}_{2} \mathrm{~S}$ (MW 584); crystals; $70 \%$ yield; m.p ${ }^{\circ} \mathrm{C}$. Anal Calc: C, 69.84; H, 4.83; N, 5.47; S, 5.48. Found: C, 69.65; H, 4.58; N, 14.18; S, 5.26. FT-IR $\left(\mathrm{KBr} \mathrm{cm}{ }^{-1}\right) v$ max: 3485

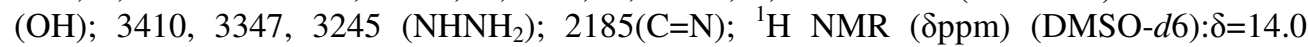
$(\mathrm{s}, 1 \mathrm{H}, \mathrm{OH}) ; 13.3(\mathrm{~s}, 1 \mathrm{H}, \mathrm{NH}) ; 7.1-7.6(\mathrm{~m}, 13 \mathrm{H}, \mathrm{Ar}-\mathrm{H}) ; 6.9(\mathrm{~s}, 1 \mathrm{H}, \mathrm{CH}) ; 5.3(\mathrm{~s}, 1 \mathrm{H}-\mathrm{CH}) ; 4.0,4.5$ $\left(2 \mathrm{br}, 4 \mathrm{H}, 2 \mathrm{xNH}_{2}\right) ; 1.5-2.9\left(\mathrm{~m}, 6 \mathrm{H}, 3 \times \mathrm{CH}_{2}\right)$. MS: $\mathrm{m} / \mathrm{z}(\%) 584\left(4 \mathrm{M}^{+}\right) ; 125(100) .{ }^{13} \mathrm{C}-\mathrm{NMR}$ (400MHz,DMSO-d6), 22.3 (CH-Pyran); 24.3, 26.0, $27.0\left(3 \mathrm{xCH}_{2}\right), 57.0$ (CH-Pyrimidine); 111-174 (aromatic $>\mathrm{C}=\mathrm{C}<) ; 117.8(>\mathrm{C} \equiv \mathrm{N}) ; 124.9,140.0(\mathrm{CH}=\mathrm{C}-$ Benzylidine $) ; 157.0(\mathrm{C}-$ $\mathrm{OH}$ ); 157.8, 164.7 (hetero aromatic $>\mathrm{C}=\mathrm{N}-$ ); $150.4,174.8$ (hetero aromatic $>\mathrm{C}-\mathrm{O}-\mathrm{C}-\mathrm{N}$ ).

\section{Antioxidant activity}

Freshly prepared $(0.004 \% \mathrm{w} / \mathrm{v})$ methanol solution of 2,2-diphenyl-1-picrylhydrzyl(DPPH) radical was prepared and stored at $10{ }^{\circ} \mathrm{C}$ in the dark. A methanol solution of the test compound was prepared. A $40 \mu \mathrm{L}$ aliquot of the methanol solution was added to $3 \mathrm{~mL}$ of DPPH solution. Absorbance measurements were recorded immediately with a UV-Visible spectrophotometer (Milton Roy, Spectronic 1201). The decrease in absorbance at $515 \mathrm{~nm}$ was determined continuously, with data being recorded at $1 \mathrm{~min}$ intervals until the absorbance 
stabilized (16 min). The absorbance of the DPPH radical without antioxidant (control) and the reference compound ascorbic acid were also measured. All the determinations were preformed in three replicates and averaged. The percentage inhibition (PI) of the DPPH radical was calculated according to the formula:

$$
\mathrm{PI}=[[(\mathrm{AC}-\mathrm{AT}) / \mathrm{AC}] \mathrm{x} 100]]
$$

Where $\mathrm{AC}=\mathrm{Absorbance}$ of the control at $\mathrm{t}=0 \mathrm{~min}$ and $\mathrm{AT}=\mathrm{Absorbance}$ of the sample + $\mathrm{DPPH}$ at $\mathrm{t}=16 \mathrm{~min}$.

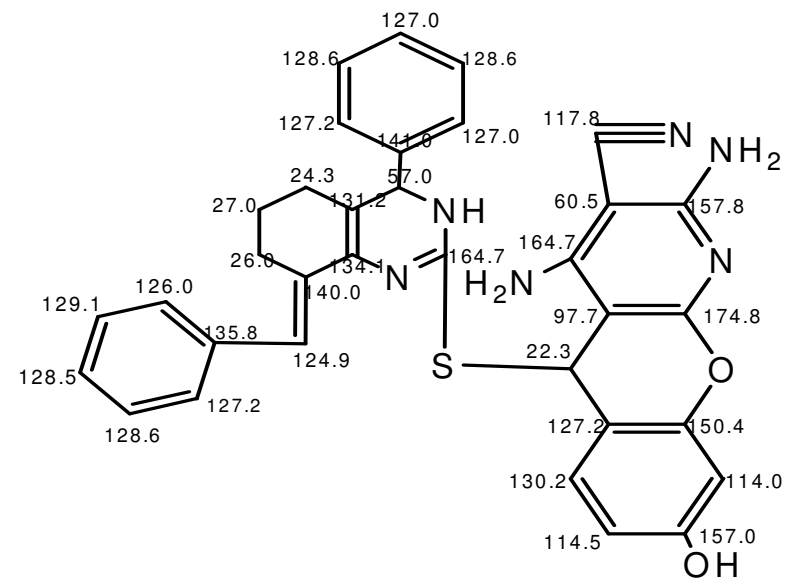

Figure 1. Structural assignment of typical carbon in compound 19 by ${ }^{13} \mathrm{C}$ NMR

\section{Results and Discussion}

In the present work, the synthetic strategies utilized for the synthesis of the target compounds are outlined in (Schemes 1-5) which could assemble entirely the desired quinazoline derivatives (2-19) from the strategic starting materials 4-aryl-8-arylidene3,4,5,6,7,8-hexahydroquinazoline-2-thiol 1a-c which was prepared in accordance with the method described in the literature ${ }^{21}$. Hence, hydrazinolysis of 8-arylidene-4-phenyl3,4,5,6,7,8-hexahydroquinazoline-2-thiol derivative $\mathbf{1 a}^{22}$ afforded hydrazinoquinazoline 2 . The structure of compound $\mathbf{2}$ was elucidated on the basis of elemental analysis, spectral data and chemical transformation. FT-IR spectrum showed an absorption bands at 3411, 3338, $3285 \mathrm{~cm}^{-1}$ attributable to $\mathrm{NHNH}_{2}$, in addition devoid the presence of thione - thiol. Its ${ }^{1} \mathrm{HNMR}$ spectrum exhibited the characteristic a broad $\mathrm{D}_{2} \mathrm{O}$ exchangeable singlets at $\delta$ 4.7, 8.7, $10.2 \mathrm{ppm}$ due to $\mathrm{NH}_{2}$ and $\mathrm{NH}$ protons, respectively.

The hydrazine analogue 2 was used as a precursor for the synthesis of condensed and non condensed quinazoline. Thus, the reaction of 2 with $\mathrm{AC}_{2} \mathrm{O}$ afforded 1-(9-arylidene-3methyl-5-aryl-6,7,8,9-tetrahydro[1,2,4]triazolo[3,4-b]quinazolin-1(5H)-yl)ethanone $\mathbf{3}$ on acetylation followed by cyclization ${ }^{23}$. The structure of compound $\mathbf{3}$ was characterized by FTIR spectrum which showed the disappearance of the characteristic bands for $\mathrm{NHNH}_{2}$ groups and appearance of absorption band at $1736 \mathrm{~cm}^{-1}$ corresponding to carbonyl acetyl group. The ${ }^{1} \mathrm{H}$ NMR spectrum revealed two singlets of six protons at $\delta 1.2,2.5 \mathrm{ppm}$ for $2 \mathrm{CH}_{3}$ groups. Condensation of compound 3 with thiosemiarbazide yielded the corresponding 1-(1-(9arylidene-3-methyl-5-phenyl-6,7,8,9-tetrahydro-[1,2,4] triazolo [3,4- $b]$ quinazolin-1 $(5 H)$ yl)ethylidene)thiosemicarb- azide 4. FT-IR spectrum showed absorption bands at 3421, 3235,3125 and $1274 \mathrm{~cm}^{-1}$ attributable to $\mathrm{NHNH}_{2}$ and $\mathrm{C}=\mathrm{S}$ groups, respectively. Its ${ }^{1} \mathrm{H}$ NMR 
spectrum showed two singlets of three protons at $\delta 8.9,5.4 \mathrm{ppm}$ for $\mathrm{NH}$ and $\mathrm{NH}_{2}$ groups. The structure of compound $\mathbf{4}$ was confirmed chemically by the reaction with aromatic aldehydes namely, 2-chlorobenzaldehyde to give the corresponding Schiff's base 5. FT-IR spectrum of 5 showed absorption bands at 3248, 1117, $611 \mathrm{~cm}^{-1}$ for $\mathrm{NH}, \mathrm{C}=\mathrm{S}$ and $\mathrm{C}-\mathrm{Cl}$ groups, whereas the mass spectrum revealed molecular ion peak $\mathrm{m} / z$ at $591.5\left(2 \% \mathrm{M}^{+}\right)$with base peak $m / z$ at $60(100 \%)$.

On the other hand, condensation of $\mathbf{3}$ with 2-chlorobenzaldehde under Claisen-Schmidt conditionsafforded1-(9-arylidene-3-methyl-5-phenyl-6,7,8,9-tetrahydro-[1,2,4]triazolo[3,4- $b$ ] quinazolin-1 $(5 H)$-yl)-3-(2-chlorophenyl)prop-2-en-1-one 6 which was reacted with thiosemicarbazide to afford 3-(9-arylidene-3-methyl-5-phenyl-6,7,8,9-tetrahydro-[1,2,4]triazolo[3,4-b] quinazolin-1(5H)-yl)-5-(2-chlorophenyl)-4,5-dihydropyrazole-1-carbothioamide 7.

The reaction takes place via Aza-Michael addition to the unsaturated carbonyl moiety followed by cyclocondensation ${ }^{22}$. FT-IR spectrum of $\mathbf{6}$ showed strong absorption band at $1667 \mathrm{~cm}^{-1}$ indicated the presence of $\alpha, \beta$-unsaturated carbonyl group, whereas ${ }^{1} \mathrm{H} \mathrm{NMR}$ spectrum exhibited the characteristic two doublet signals of two protons at $\delta 6.6,7.8 \mathrm{ppm}$ for $\mathrm{COCH}=\mathrm{CH}$. The structure of 7 was characterized by FT-IR spectrum which showed absorption bands at 3421,3385 and $1274 \mathrm{~cm}^{-1}$ attributable to $\mathrm{NH}_{2}$ and $\mathrm{C}=\mathrm{S}$, respectively, whereas the ${ }^{1} \mathrm{H}$ NMR spectrum revealed the ring protons (HA and $\mathrm{HB}$ ) as a pair of doublet near $3.3 \mathrm{ppm}$ and also vicinal methane proton $(1 \mathrm{H}, \mathrm{CH}$ of pyrazoline) showed triplet near $4.3 \mathrm{ppm}$ due to vicinal coupling with the two magnetically non equivalent protons of the methylene group $\mathrm{HA}$ (up field $\mathrm{H}$ of $\mathrm{CH}_{2}$ ) and $\mathrm{HB}$ (downfield $\mathrm{H}$ of $\mathrm{CH}_{2}$ ) and singlet signal at $4.6 \mathrm{ppm}$ for $\mathrm{NH}_{2}$ protons.

The reaction of 2-chlorobenzaldehyde with malononitrile under Knoevenagel condensation conditions afforded the Michael acceptor which reacted with compound $\mathbf{3}$ in the presence of ammonium acetate to give 3-amino-6-(9-arylidene-3-methyl-5-phenyl-6,7,8,9-tetrahydro-[1,2,4] triazolo[3,4-b]quinazolin-1(5H)-yl)-4-(4-chlorophenyl)picolinonitrile $\mathbf{8}^{24}$ (Scheme 1).

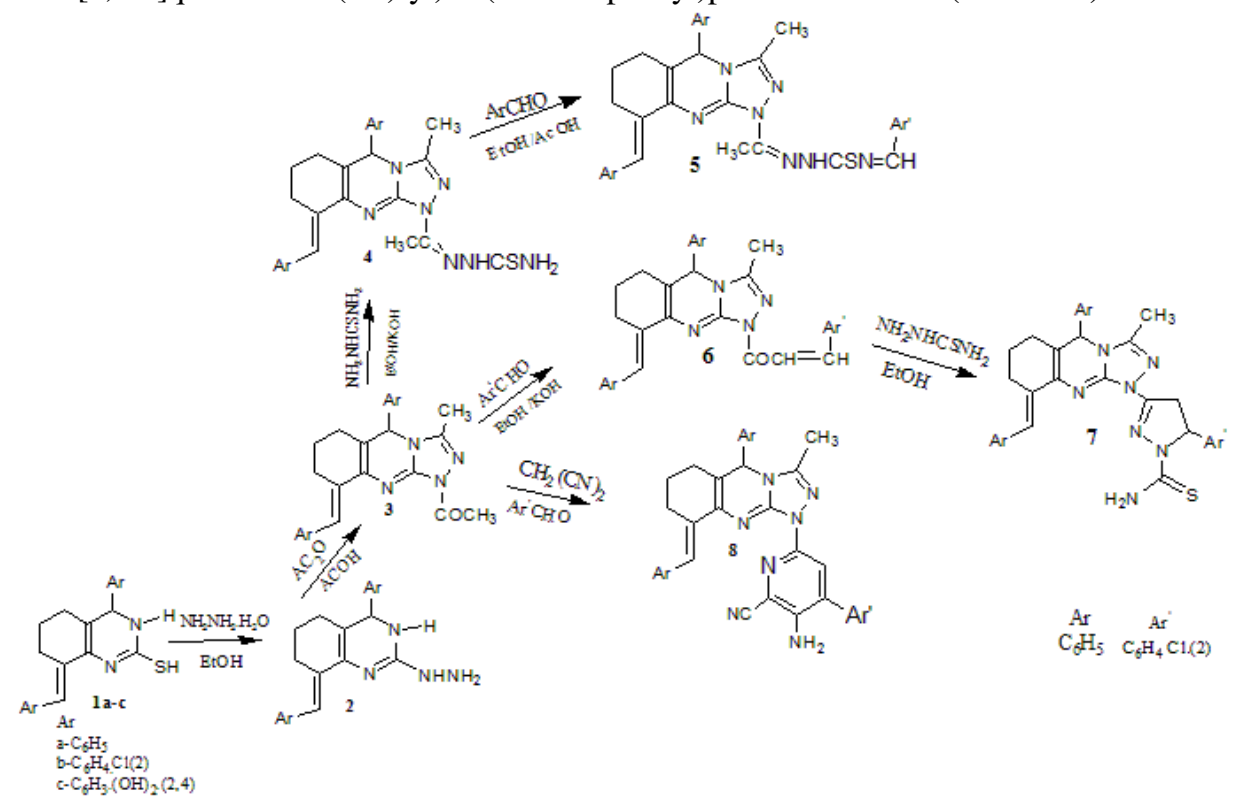

Scheme 1 
Reaction of compound 2 with diethyl oxalate in the presence of sodium ethoxide yielded the unexpected ethyl-2-(2-(8-arylidene-4-phenyl-3,4,5,6,7,8-hexahydroquinazolin-2yl) hydra- zinyl)-2-oxo-acetate 9 not the expected compound ${ }^{25}$ (Scheme 2).
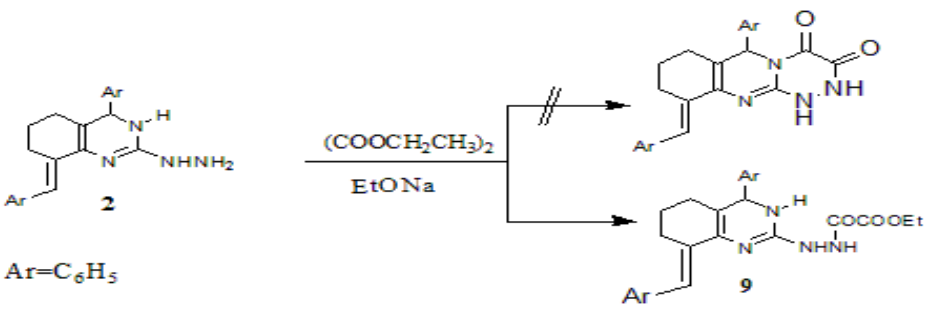

Scheme 2

The FT-IR spectrum of the latter compound showed bands at 3215,1741 and $1672 \mathrm{~cm}^{-1}$ attributable to $\mathrm{NH}, \mathrm{CO}$ groups whereas the ${ }^{1} \mathrm{HNMR}$ spectrum displayed a triplet of three protons at $\delta 1.2 \mathrm{ppm}$ for methyl group, a quartet of two protons at $\delta 4.1 \mathrm{ppm}$ for methylene group and three singlet protons at $\delta 14.2,12.4$ and $\delta 10.0 \mathrm{ppm}$ due to $\mathrm{CONH}$ and two $\mathrm{NH}$ groups. The ${ }^{13} \mathrm{C}$-NMR spectrum of compound 9 showed the presence of signals at $\delta$ : 15.6 $\left(\mathrm{CH}_{3}\right), 63.8\left(\mathrm{CH}_{2}\right), 56.5$ (CH-Pyrimidine), 163.9, $165.2(2 \mathrm{C}=\mathrm{O}), 160.1(\mathrm{C}-\mathrm{NH}-\mathrm{NH})$ 122.0, 137.3 ( $\mathrm{CH}=\mathrm{C}$-benzylidine). Also, condensation of compound 2 with ethyl acetoacetate in acetic acid didn't yield the expected compound according to literature ${ }^{26}$ and gave 3-(8-aeylidene-4-phenyl3,4,5,6,7,8-hexahydroquinazolin-2-yl)-3-oxo-butanehydrazide $\mathbf{1 0}$ (Scheme 3).

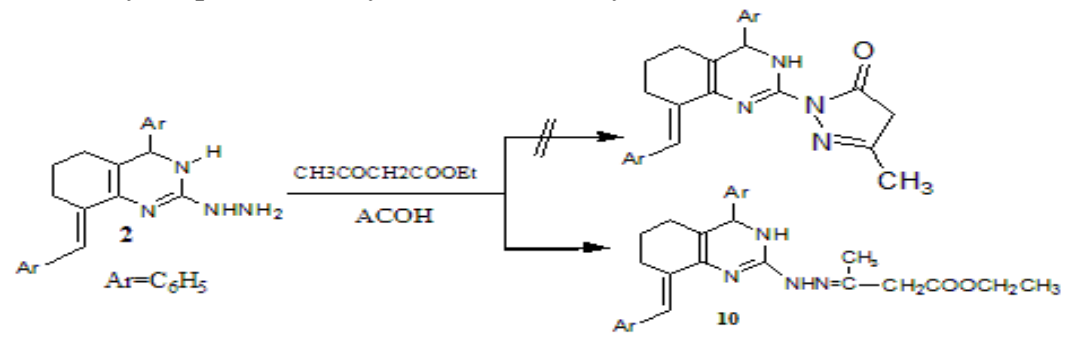

Scheme 3

The FT-IR spectrum of $\mathbf{1 0}$ showed absorption bands at 3232, 1716, $1670 \mathrm{~cm}^{-1}$ indicated the presence of $\mathrm{NH}, \mathrm{CO}$ ester and amide whereas, the ${ }^{1} \mathrm{HNMR}$ spectrum revealed two singlets at $\delta 12.0,10.5 \mathrm{ppm}$ for two $\mathrm{NH}$ protons, a triplet of three protons at $\delta 1.4 \mathrm{ppm}$ for $\mathrm{CH}_{3}$ group, a quartet at $\delta 4.8 \mathrm{ppm}$ for $\mathrm{CH}_{2} \mathrm{COO}$ protons, and a singlet signal at $\delta 3.9 \mathrm{ppm}$ for $\mathrm{CH}_{2}$ protons, respectively.

Treatment of 2 with $\mathrm{CS}_{2}$ in boiling pyridine afforded the corresponding 9-arylidene-5phenyl-1,2,6,7,8,9-hexahydro-[1,2,4]triazolo[3,4- $b]$ quinazoline-3(5H)-thione $\mathbf{1 1}$ [23,27]. FT-IR spectrum of $\mathbf{1 1}$ showed disappearance of $\mathrm{NH}_{2}$ and appearance of an absorption band at 1267 indicated the presence of $(\mathrm{C}=\mathrm{S})$. Its ${ }^{1} \mathrm{H}$ NMR spectrum showed two singlets at $\delta$ $12.0,10.5 \mathrm{ppm}$ for $2 \mathrm{NH}$ protons, in addition to disappearance of $\mathrm{NH}_{2}$ protons (Scheme 4).

Mixing equimolecular amounts of compound 2, malononitrile and 2-chlorobenzaldehyde in boiling ethanol and catalytic amount of pyridine has produced the corresponding 3-amino11-arylidene-5-(2-chlorophenyl)-7-phenyl-1,4,5,7,8,9,10,11-octahydro-[1,2,4]triazepino[3,4b] quinazoline-4-carbonitrile 12 (Scheme 4). The chemical structure of 12 was elucidated on the basis of spectral techniques. The FT-IR spectrum showed the characteristic absorption bands at 3362, 3276, 3150, $2199 \mathrm{~cm}^{-1}$ for $\mathrm{NH} \mathrm{NH}_{2}$ and $\mathrm{C} \equiv \mathrm{N}$, whereas the ${ }^{1} \mathrm{H} \mathrm{NMR}$ displayed 
singlet signals of one proton at $\delta 6.9 \mathrm{ppm}$ for $\mathrm{NH}$ group, two doublet of two protons at $\delta 3.2$, $3.9 \mathrm{ppm}$ for $(2 \times \mathrm{CH}$ triazepine $)$ and one singlet broad of two protons at $\delta 4.6 \mathrm{ppm}$ indicated the presence of $\mathrm{NH}_{2}$ group. The mass spectrum of $\mathbf{1 2}$ showed molecular ion peak at $\mathrm{m} / \mathrm{z}, 519$ $\left(\mathrm{M}^{+} 3 \%\right)$, with base peak $\mathrm{m} / \mathrm{z}$ at $257(100 \%)$. On the other hand, compound 1 was reacted with chloroacetyl chloride in boiling pyridine to yield the corresponding $13^{28}$ which used as a key for the synthesis of a series of heterocyclic compounds. Structure of $\mathbf{1 3}$ could however be assigned to the reaction product based on FT-IR spectrum which indicated the presence of carbonyl group at $1712 \mathrm{~cm}^{-1}$ whereas, the ${ }^{1} \mathrm{H}$ NMR revealed a singlet of two protons at $\delta$ $4.3 \mathrm{ppm}$ for $\mathrm{CH}_{2}$ group. The acylation of appropriate cyclic ketones $\mathbf{1 3}$ with diethyl oxalate gave $\alpha, \gamma$-diketoester 14, which was reacted with hydrazine provided the bicyclic pyrazole esters 15 and the bicyclic pyrazole hydrazide 16 (Scheme 5$)^{29}$. The structure of 14 was supported by the FT-IR spectrum which showed absorption band at $1728 \mathrm{~cm}^{-1}$ assigned to carbonyl of ( $\alpha$-keto ester) and its ${ }^{1} \mathrm{H}$ NMR which revealed a singlet of one proton at $\delta 4.3$ ppm for $\mathrm{CH}$-thiazole, a triplet of three protons at $\delta 1.1 \mathrm{ppm}$ for $\mathrm{CH}_{3}$ group, a quartet of two protons at $\delta 4.1 \mathrm{ppm}$ for $\mathrm{CH}_{2}$ group, respectively. The mass spectrum of 14 showed molecular ion peak at $m / z 472\left(\mathbf{M}^{+} 6 \%\right)$, with base peak $m / z$ at $125(100 \%)$.

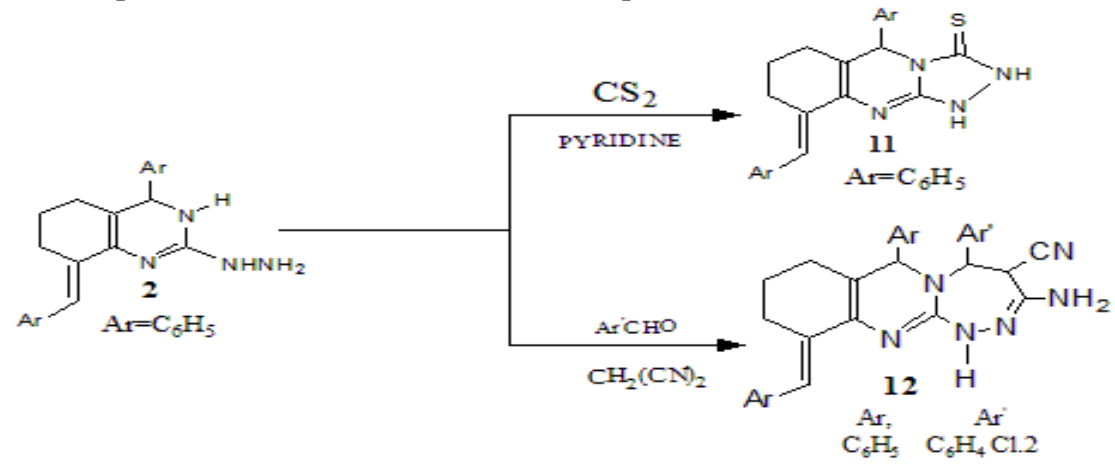

Scheme 4

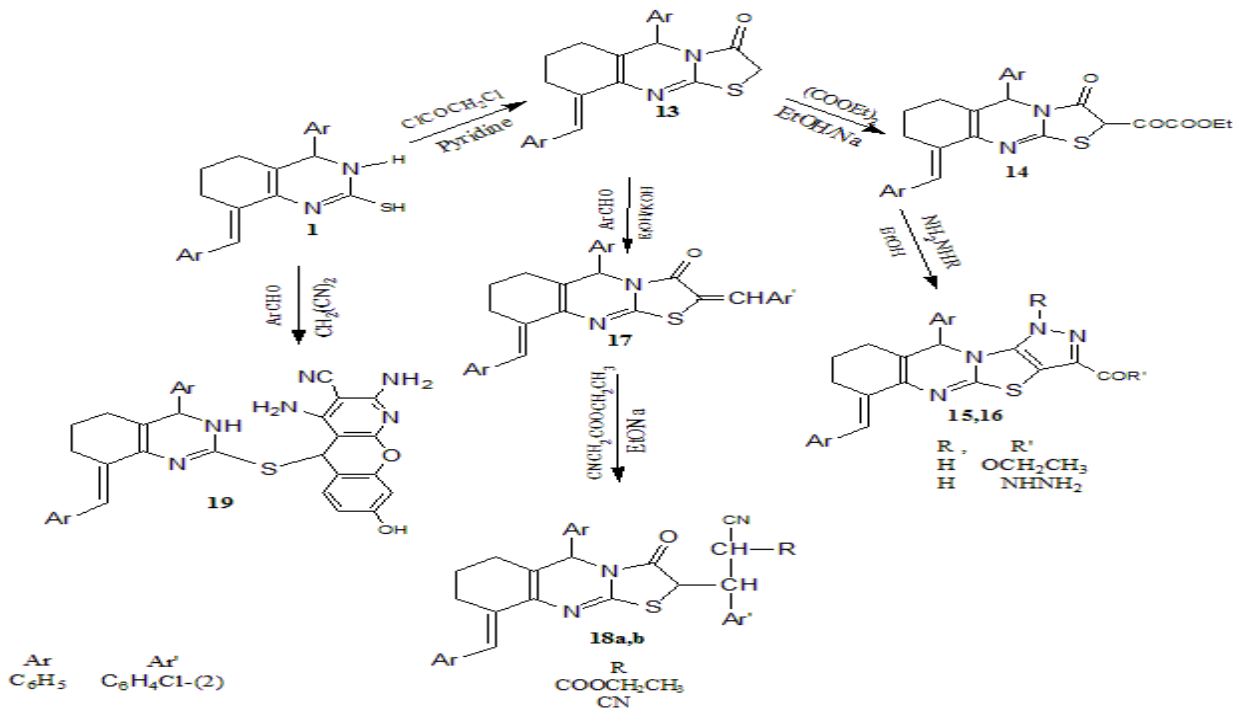

Scheme 5 
The analytical and spectral data of $\mathbf{1 5}$ and $\mathbf{1 6}$ were in agreement with the proposed structures. ${ }^{1} \mathrm{H}$ NMR spectrum of $\mathbf{1 5}$ showed a singlet of one proton at $\delta 8.1 \mathrm{ppm}$ for $\mathrm{NH}$ group, a quartet of two protons at $\delta 4.2 \mathrm{ppm}$ for methylene group, a triplet of three protons at $\delta 1.2 \mathrm{ppm}$ for methyl group. The mass spectrum of $\mathbf{1 5}$ showed molecular ion peak at 468 $\left(\mathrm{M}^{+} 11 \%\right)$ with base peak $\mathrm{m} / \mathrm{z}$ at $343(100 \%)$, whereas, the ${ }^{1} \mathrm{H}$ NMR spectrum of 16 revealed two singlets of two protons at $\delta 10.6,9.9 \mathrm{ppm}$ for two $\mathrm{NH}$ groups, a broad signal of two protons at $\delta 4.2 \mathrm{ppm}$ for $\mathrm{NH}_{2}$ group and devoid the signals of ester protons. Condensation of 13 with aromatic aldehydes in boiling ethanol under Claisen-Schmidt conditions gave 2-(2chlorobenzylidene)-9-arylidene-5-phenyl-6,7,8,9-tetrahydro- $2 H$-thiazolo $\quad[2,3-b]$ quinazolin$3(5 H)$-one 17 which was reacted with active methylene compounds namely, ethyl acetoactate and /or malononitrile to afford ethyl 3-(9-arylidene-3-oxo-5-phenyl-3,5,6,7,8,9hexahydro-2 $H$-thiazolo[2,3-b]quinazolin-2-yl)-3-(2-chlorophenyl)-2-cyanopropanoate (18a) and 2-(9-arylidene-3-oxo-5-phenyl-3,5,6,7,8,9-hexahydro- $2 H$-thiazolo[2,3-b]quinazolin-2yl)(2-chlorophenyl)methyl) malononitrile (18b), respectively. The ${ }^{1} \mathrm{H}$ NMR spectrum of 18a showed a multiplet of three proton at 3.7-3.9 for three methine groups, a quartet of two protons at $\delta 4.1 \mathrm{ppm}$ for methylene group, a triplet of three protons at $\delta 1.2 \mathrm{ppm}$ for methyl group whereas, the ${ }^{1} \mathrm{H}$ NMR spectrum of $\mathbf{1 8 b}$ showed a multiplet of three proton at 3.2-3.9 ppm for three methine groups.

On the other hand, compound $\mathbf{1}$ was reacted with malononitrile and aromatic aldehydes namely, 2,4-dihydroxybenzaldehyde in a ratio 1: $2: 1$ to give 2,4-diamino-5-(8-arylidene-4phenyl-3,4,5,6,7,8-hexahydroquinazolin-2-yl-thio)-5H-chromeno[2,3- $b$ ]pyridine-3 carbonitrile $1^{30}$. The analytical and spectral data of 19 was in agreement with the proposed structure. The FT-IR spectrum of 19 showed absorption bands at 3485, 3410, 3347, 3245 and 2185 attributable to $(\mathrm{OH}),\left(\mathrm{NHNH}_{2}\right)$ and $(\mathrm{C} \equiv \mathrm{N})$. Its ${ }^{1} \mathrm{H}$ NMR revealed the presence of one singlet at $\delta 14.0 \mathrm{ppm}$ of one proton for $(\mathrm{OH})$ group, one singlet of one proton at $\delta 13.2 \mathrm{ppm}$ for $(\mathrm{NH})$ and two broad signal of four protons at $\delta 4.0,4.5 \mathrm{ppm}$ for two $\left(\mathrm{NH}_{2}\right)$ groups, whereas the mass spectrum showed molecular ion peak at $m / z, 584\left(\mathrm{M}^{+} 4 \%\right)$ with base peak at $m / z, 125(100 \%)$.

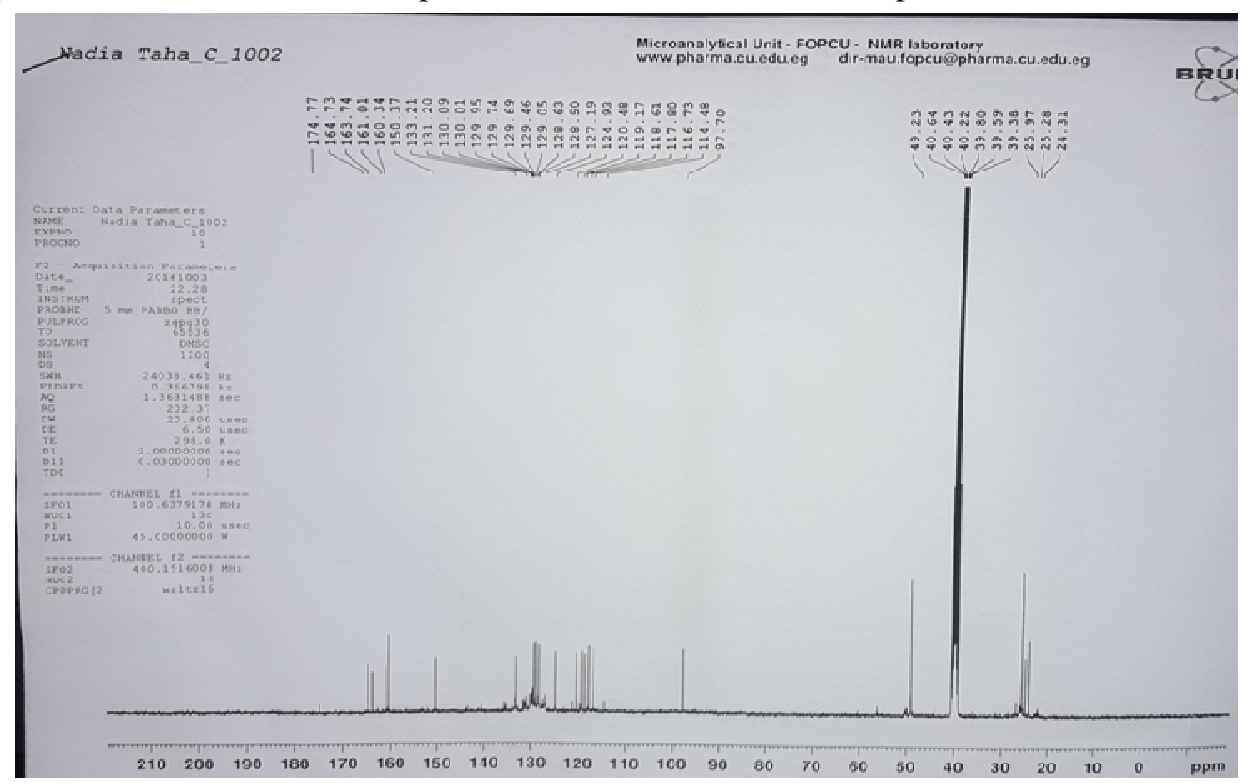

Figure 2. ${ }^{13} \mathrm{C}$ NMR Spectrum of compound 19 
The ${ }^{13} \mathrm{C}$ NMR spectrum (Figure 2) of compound 19 selected as a prototype showed the presence of signals at $\delta: 22.3(\mathrm{CH}-\mathrm{Pyran}), 57.0(\mathrm{CH}-\mathrm{Pyrimidine}), 117.8(>\mathrm{C} \equiv \mathrm{N}), 124.9$, $140.0(\mathrm{CH}=\mathrm{C}-$ Benzylidine $), 157.0(\mathrm{C}-\mathrm{OH})$.

\section{Pharmacology}

\section{Antioxidant activity}

The free radical scavenging activity of all the tested compounds was evaluated according to the method described in the literature ${ }^{31,32}$. The inhibition of all the tested synthesized compounds on (1,1-diphenyl-2-picrylhydrazyl) DPPH free radical by using ascorbic acid (AA) antioxidant agent as positive control in different concentrations are listed in Table 1. The antioxidant activity is expressed in terms of $\%$ inhibition and $\mathrm{IC}_{50}$ (effective concentration for scavenging $50 \%$ of the initial DPPH) value $(\mu \mathrm{M})$.

Table 1. Antioxidant activity of the new synthesized compounds (\% inhibition)

\begin{tabular}{cccccccccc}
\hline \multirow{2}{*}{$\begin{array}{c}\text { Comp. } \\
\text { Code }\end{array}$} & \multicolumn{10}{c}{ Concentration, $\mu \mathrm{g} / \mathrm{mL}$} & \multirow{2}{*}{$\mathrm{IC}_{50}$} \\
\cline { 2 - 9 } & 2 & 4 & 8 & 16 & 32 & 64 & 128 & 356 & $\mu \mathrm{g} / \mathrm{mL}$ \\
\hline 2 & 16.52 & 24.26 & 41.13 & 56.09 & 67.13 & 81.57 & 82.78 & 84.61 & 12.7 \\
3 & 16.43 & 25.13 & 40.65 & 51.00 & 60.00 & 71.65 & 80.96 & 83.57 & 6.35 \\
5 & 14.20 & 20.60 & 28.60 & 39.60 & 52.60 & 83.70 & 84.70 & 85.80 & 144 \\
12 & 11.91 & 20.52 & 29.65 & 41.57 & 60.78 & 74.09 & 82.43 & 84.43 & 23.02 \\
13 & 4.17 & 8.00 & 21.48 & 27.22 & 32.78 & 43.65 & 59.04 & 75.30 & 90.4 \\
15 & 18.43 & 29.13 & 41.65 & 52.00 & 64.00 & 73.65 & 80.96 & 83.57 & 7.22 \\
16 & 18.96 & 20.96 & 29.22 & 36.35 & 45.57 & 68.70 & 82.78 & 86.70 & 19.06 \\
19 & 11.65 & 15.39 & 26.00 & 36.83 & 59.39 & 73.13 & 81.65 & 83.65 & 24.3 \\
\hline Standard & \multicolumn{10}{c}{ Concentration, $\mu \mathrm{g} / \mathrm{mL}$} & & & \\
\hline Ascorbic & 5 & 10 & 15 & 20 & 25 & 30 & 35 & 40 & $\mathrm{IC}_{50} \mu \mathrm{g} / \mathrm{mL}$ \\
acid & 12.98 & 16.38 & 62.98 & 76.81 & 78.72 & 78.94 & 80.21 & 86.36 & 11.2 \\
\hline
\end{tabular}

Of all the compounds tested synthesized show that compounds $2,3, \mathbf{5}, \mathbf{1 2}, \mathbf{1 3}, \mathbf{1 5}, 16$ and 19 showed scavenging activity towards DPPH. Compounds 3 and 15 showed very strong inhibition than the specific inhibitor AA. Compounds 2, 12, 16 and 19 showed good inhibition whereas, compounds $\mathbf{5}, \mathbf{1 3}$ showed moderate to weak inhibition, as shown in Figures $3 \& 4$.

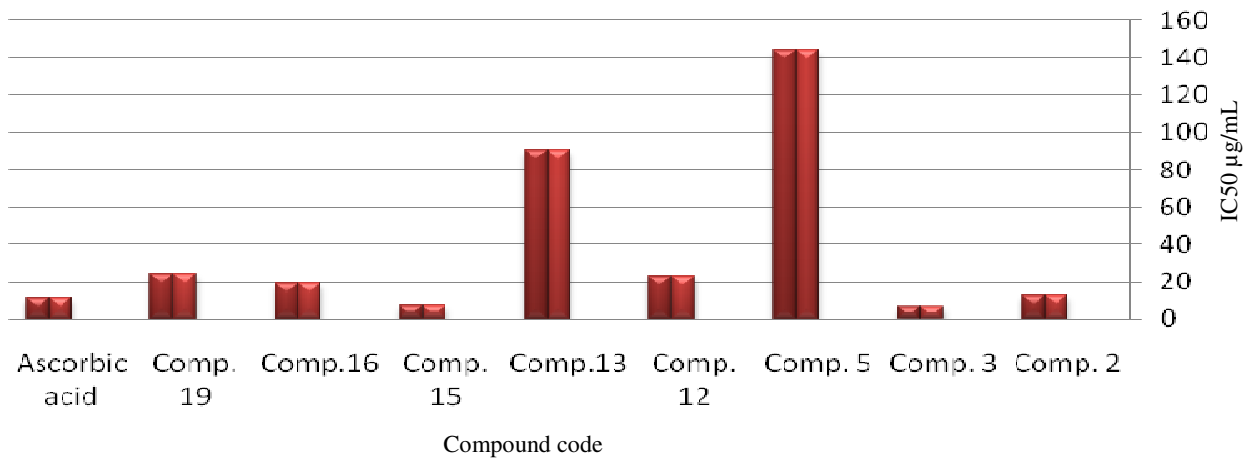

Figure 3. Antioxidant activity of the new synthesized compounds (\% inhibition) compared to ascorbic acid as a standard compound 

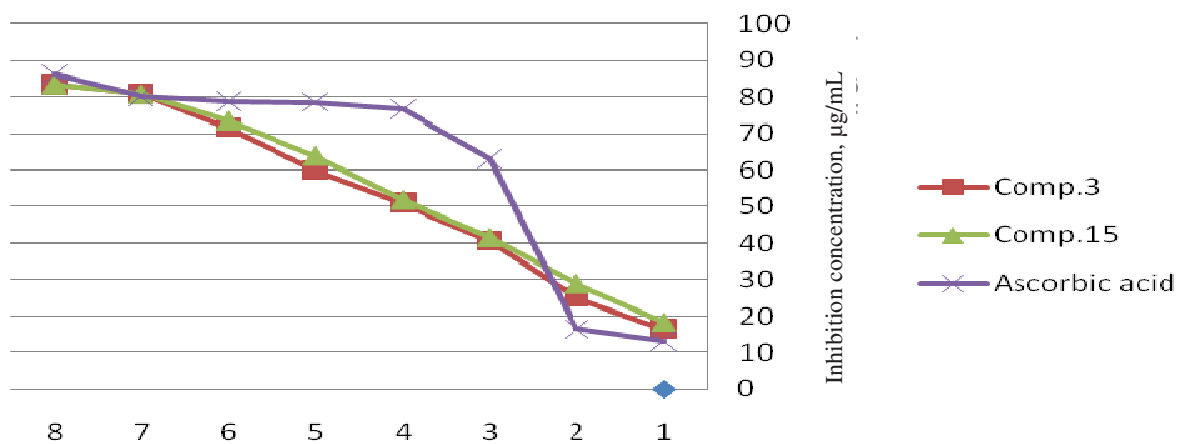

Figure 4. Antioxidant activity of compounds $\mathbf{3}$ and $\mathbf{1 5}$ (\% inhibition) compared to ascorbic acid as a standard compound

The higher inhibition of compound $\mathbf{3}$ is due to the conjugate free radicals on the methylene carbon atom with the carbonyl group (due to the elimination of hydrogen proton from methyl group). The suggested mechanism for the reaction of compounds 3 with DPPH radical is presented in Scheme 6.

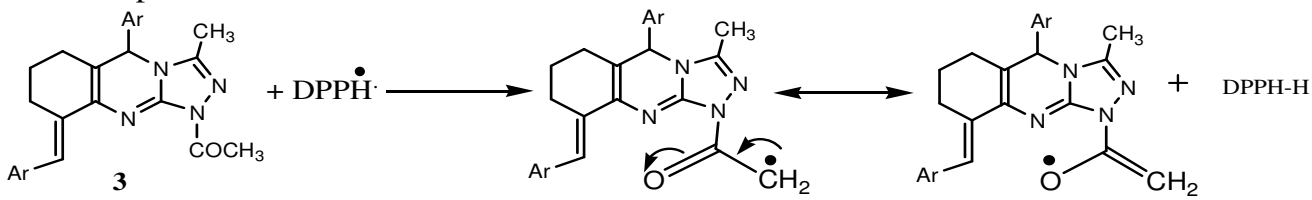

$\mathrm{Ar}=\mathrm{C}_{6} \mathrm{H}_{5}$

\section{Scheme 6}

Whereas, the higher inhibition of compound $\mathbf{1 5}$ is due to the conjugate free radicals on the nitrogen atom of pyrazole derivative with carbonyl ester group (due to the elimination of hydrogen proton from nitrogen atom of NH-pyrazole). The suggested mechanism for the reaction of compounds $\mathbf{1 5}$ with DPPH radical is presented in Scheme 7.

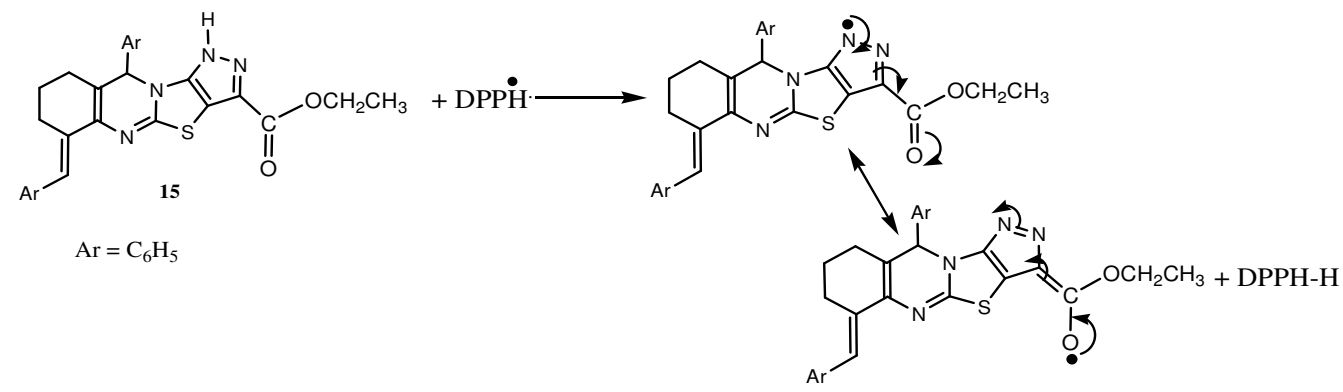

Scheme 6

\section{Conclusion}

In the investigation described above, the 4-aryl-8-arylidene-3,4,5,6,7,8-hexahydroquinazoline -2-thiol (1) moiety was introduced as a new class of antioxidant agent. The scaffold has the advantage of facile synthetic protocol access. Briefly, target compounds were prepared via reactions of 8-arylidene-4-phenyl-3,4,5,6,7,8-hexahydroquinazoline-2-thiol (1a) and 1-(8- 
arylidene-4-phenyl-3,4,5,6,7,8-hexahydroquinazolin-2-yl)hydrazine (2) with nucleophilic and electrophilic reagents. The new synthesized products were found to exhibit antioxidant activities compared to ascorbic acid as reference. Among all the test compounds, the most reactive compounds were $\mathbf{3}$ and $\mathbf{1 5}$.

\section{References}

1. Chandragowda V, Kush A K and Chandrasekara Reddy G, Eur J Med Chem., 2009, 44(7), 3046-3055; DOI:10.1016/j.ejmech.2008.07.023

2. Al-Rashood S T, Aboldahab I A, Nagi M N, Abou Zeid L A, Abdel-Aziz A A, AbdelHamide S G, Youssef KM, Al-Obaid A M and El-Subbagh H, Bioorg Med Chem; 2006, 14(24), 8608-8621; DOI:10.1016/j.bmc.2006.08.030

3. Vasdev N, Dorff P N, Gibbs A R, Nandanan E, Reid L M, Neil J P O and Van Brocklin H F, J Labeled Comp Rad., 2005, 48(2), 109-115; DOI:10.1002/jlcr.903

4. Wakeling A E, Guy S P, Woodburn J R, Ashton S E, Curry B J, Barker A J and Gibson K H, Cancer Res., 2002, 62(20), 5749-5754.

5. Alagarsamy V, Solomon V R and Dhanabal K, Bioorg Med Chem., 2007, 15(1), 235241; DOI:10.1016/j.bmc.2006.09.065

6. Baba A, Kawamura N, Makino H, Ohta Y, Taketomi S and Sohda T, J Med Chem., 1996, 39(26), 5176-5182; DOI:10.1021/jm9509408

7. Rohini R, Muralidhar Reddy P, Shanker K, Hu A and Ravinder V, Eur J Med Chem., 2010, 45(3), 1200-1205; DOI:10.1016/j.ejmech.2009.11.038

8] Antipenko L, Karpenko A, Kovalenko S, Katsev A, Komarovska-Porokhnyavets E, Novikov V and Chekotilo A, Chem Pharm Bull., 2009, 57(6), 580-585.

9. Jatav V, Kashaw S and Mishra P, Med Chem Res., 2008; 17(2), 205-211; DOI:10.1007/s00044-007-9054-3.

10. Aly A A, Chin J Chem., 2003, 21(3), 339-346; DOI:10.1002/cjoc.20030210324

11. Li H, Huang R, Qiu D, Yang Z, Liu X, Ma J and Ma Z, Prog Nat Sci., 1998, 8, 359-365.

12. Chandrika P M, Yakaiah T, Narsaiah B, Sridhar V, Venugopal G, Rao J V, Kumar K P, Murthy U S N and Rao A R R, Indian J Chem., 2009, 48B, 840-847.

13. Paneersalvam P, Raj T, Ishar P S M, Singh B, Sharma V, Rather B A, Indian J Pharm Sci., 2010, 72(3), 375-378.

14. Nandy P, Vishalakshi M T and Bhat A R, Indian J Heterocycl Chem., 2006, 15, 293-294.

15. Saravanan G, Alagarsamy V and Prakash C R, Int J Pharm Pharm Sci., 2010, 2(4), 83-86.

16. Lakhan R, Singh O P and Singh J R L, J Indian Chem Soc., 1987, 64, 316-318.

17. Hess H J, Cronin T H and Scriabine A, J Med Chem., 1968, 11(1), 130-136;

DOI:10.1021/jm00307a028

18. Sasmal S, Balaji G, Kanna Reddy H R, Balasubrahmanyam D, Srinivas G, Kyasa S, Sasmal P K, Khanna I, Talwar R, Suresh J, Jadhav V P, Muzeeb S, Shashikumar D, Harinder Reddy K, Sebastian V J, Frimurer T M, Rist $\varnothing$, Elster L and Högberg T, Bioorg Med Chem Let., 2012, 22(9), 3157-3162; DOI:10.1016/j.bmcl.2012.03.050

19. Alvarado M, Barceló M, Carro L, Masaguer C F and Raviña E, Chem Biodivers, 2006, 3(1), 106-117; DOI:10.1002/cbdv.200690001

20. Malamas MS and Millen J, J Med Chem., 1991, 34(4), 1492-1503; DOI:10.1021/jm00108a038.

21. Nadia T A D, Chem Sci Trans., 2013, 2(1), 129-134; DOI:10.7598/cst2013.338

22. EL-Hashasha M A, EL-Badry Y A and Morsyc J M, J Advances Chem., 2013, 5(2), 669-677. 
23. Hala B El-Nassan, Eur J Med Chem., 2011, 46(6), 2031-2036;

DOI:10.1016/j.ejmech.2011.02.055

24. Mashkouri S and Reza Naimi-Jamal M, Molecules, 2009, 14(1), 474-479; DOI:10.3390/molecules14010474

25. Saleh A Bahashwan, Ahmed A Fayed, Abd El-Galil E Amr, Eman M Flefel and Kalmouch A, Molecules, 2013, 18(2), 15051-15063; DOI:10.3390/molecules181215051

26. Ekhlass N, Yaser A El-Badry, Afaf M M Eltoukhy and Rezek R Ayyad, Med Chem (Los Angeles) 2016, 6(4), 224-233; DOI:10.4172/2161-0444.1000350

27. Ola H R, Omaima G S and Ibrahim M El-Ashmawy, Eur J Med Chem., 2012, 55, 8593; DOI:10.1016/j.ejmech.2012.07.007

28. Mosaad S M, Rehab K and Samar S F, Eur J Med Chem., 2010, 45(7), 2994-3004; DOI:10.1016/j.ejmech.2010.03.028

29. Semple G, Skinner P J, Gharbaoui T, Shin Y-J, Jung J-K, Cherrier M C, Webb P J, Tamura S Y, Boatman P D, Sage C R, Schrader T O, Chen R, Colletti S L, Tata J R, Waters M G, Cheng K, Taggart A K, Cai T-Q, Carballo-Jane E, Behan D P, Connolly D T and Richman J G, J Med Chem., 2008, 51(16), 5101; DOI:10.1021/jm800258p

30. Hai-Feng G, Wei-Wei C, Zheng F, Xin L, Shi-Gui T and Kai G, Chinese Chem Lett., 2014, 25(10), 1357-1362; DOI:10.1016/j.cclet.2014.05.008

31. Yen G C and Duh P D, J Agric Food Chem., 1994, 42(3), 629-632; DOI:10.1021/jf00039a005

32. Koparir M, Orek C, Parlak A E, Söylemez A, Koparir P, Karatepe M and Dastan S D, Eur J Med Chem., 2013, 63, 340-346; DOI:10.1016/j.ejmech.2013.02.025 